\title{
Monepantel e levamisol no controle de helmintos gastrointestinais em rebanhos ovinos no Oeste de Santa Catarina
}

Gebert, R.R. ' ; Dos Reis, J.H. '; Dos Santos, D.S. '; Boito, J.P. '; Galli, G.M. ${ }^{\text {; }}$ Baretta, D. ${ }^{1,2}$; Biazus, A.H. ${ }^{2}$; Zortéa, T. ${ }^{2}$; Favero, J.F. ${ }^{2}$ e Da Silva, A.S. ${ }^{1,2}$

'Departamento de Zootecnia. Universidade do Estado de Santa Catarina (UDESC). Chapecó. Brasil.

2Programa de Pós-graduação em Zootecnia. Universidade do Estado de Santa Catarina (UDESC). Chapecó. Brasil.

\section{PALAVRAS CHAVE ADICIONAIS}

Helmintos.

Ovinos.

Resistencia anti-helmíntica.

Antiparasitário.

Ecotoxicologia.

\section{RESUMO}

O objelivo desse estudo foi avaliar a eficácia do monepantel e levamisol sobre os helmintos gastrointestinais em rebanhos ovinos no oeste de Santa Catarina. O estudo feito em duas etapas, a primeira etapa foi selecionada cinco fazendas, e formado três grupos (K, Le M) com 8 ovinos cada. Nos animais do grupo K foi administrado monepantel $(2,5 \mathrm{mg} / \mathrm{kg}$ ), nos ovinos do grupo L foi levamisol $(5,0 \mathrm{mg} / \mathrm{kg}$ ) e os animais do grupo M foram usados como controle (não medicados). Amostras de fezes foram coletadas no dia 0 e dia 10 de experimento, analisadas pela técnica de McMaster que permite determinar o número de ovos por grama de fezes (OPG), seguido da técnica de coprocultura para identificação dos parasitos. A segunda etapa do estudo foi conduzida em uma fazenda, onde foi formado os mesmos três grupos descritos anteriormente com seus respectivos tratamentos, e cinco dias após tratamento antiparasitário foi realizada coleta de fezes desses animais para realização dos testes ecołoxicológicos, usando como marcador biológico o colêmbolo (Folsomia candida). A fim de simular o uso das fezes dos ovinos como adubo orgânico, definimos as doses $0 ; 2 ; 4 ;$ e 8 ton/ha, assim como os testes de sobrevivência e reprodução de acordo com metodologias ISO. Os animais que receberam o tratamento com monepantel fiveram redução do OPG $(\mathbb{P}<0.05)$ comparado aos demais grupos. Em apenas uma fazenda, a redução de OPG foi inferior $(\mathrm{P}<0,05)$ nos animais tratados com levamisol comparado ao grupo controle. Nos grupos tratados com monepantel foi observado a resistência do Trichostrongylus spp. ao fármaco, já o Haemonchus spp. foi resistente ao levamisol na maioria das fazendas. A eficácia do monepantel para nas cinco fazendas foi de 97, 98, 99 , 100 e 100\%, diferente do levamisol, onde nessas mesmas fazendas a eficácia foi de $69,-25,52,90$ e $50 \%$. Os ensaios ecotoxicológicos mostram que não teve diferença na sobrevivência e reprodução de colembolos entre os grupos, isto é, fezes de animais medicados com levamisol e monepantel não afeta o $F$. candida, um importante biomarcador de toxicidade do solo e impactos ambiental. Portanto, o monepantel, um produto antiparasitário relativamente novo no mercado brasileiro, pode ser a solução em fazendas de ovinos com graves problemas de resistência a antihelmínticos, pois teve eficácia desejada em todos as fazendas testadas.

\section{Monepantel and Levamisole in the control of gastrointestinal helminths in sheep herds in western Santa Catarina}

\section{SUMMARY}

\section{ADDITIONAL KEYWORDS}

Helminth.

Sheep.

Anthelmintics resistances.

Antiparasitic.

Ecotoxicology

\section{INFORMATION}

Cronología del artículo.

Recibido/Received: 18.09.2017

Aceptado/Accepted: 08.02.2018

On-line: 15.01 .2019

Correspondencia a los autores/Contact e-mail:

aleksandro_ss@yahoo.com.br
The objective of this study was to evaluate the efficacy of monepantel and levamisole on gastrointestinal helminths in sheep herds in western Santa Catarina. The study was done in two stages. In the first one, five farms were selected and three groups $(K, L$ and $M)$ of 8 sheep each were formed. In the animals of group $K$, Monepantel $(2.5 \mathrm{mg} / \mathrm{kg})$ was administered, in those of group L, Levamisole $(5.0 \mathrm{mg} / \mathrm{kg})$ and the animals of group $M$ were used as control (not medicated). The samples of feces were collected on day 0 and 10 of experiment, analyzed by the technique of McMaster with the objective of determining the number of eggs per gram of feces (HPG), followed by the technique of stool for the identification of the parasites. The second stage of the study was developed in a farm, where the same three groups were formed previously described with their respective treatments, and five days after the antiparasitic administration, feces sampling of these Animals for the performance of ecotoxicological tests, using Springtails (Folsomia candida) as a biological marker. To simulate the use of sheep feces as organic fertilizer, we define the doses 0; 24 and 8 tons of feces per hectare, as well as the survival and reproduction tests according to the ISO methodologies. The animals that received the treatment with Monepantel had reduction of the parasitic load $(P<0.05)$ compared with the remaining groups. In only one farm, the reduction of HPG was inferior $(P<0.05)$ in animals treated with levamisole compared to the control group. In the groups treated with Monepantel, resistance of Trichostrongylus spp was observed. At the same, and Haemonchus spp. It was resistant to levamisole on most farms. The efficacy of Monepantel for the five farms was $97,98,99,100$ and $100 \%$, respectively, unlike the Levamisole, where they found efficiencies of $69,25,52,90$ and $50 \%$. The ecotoxicological tests show that there was no difference in the survival and reproduction of the collectors between the groups, ie the feces of animals medicated with levamisole and Monepantel does not affect F. candida. Therefore, the Monepantel, a relatively new antiparasitic product in the Brazilian market, can be the effective alternative in sheep farms with serious problems of resistance to anthelmintics, since it had desired efficacy in all the analyzed farms. 


\section{INTRODUÇÃO}

A ovinocultura está presente em grande parte do estado de Santa Catarina (SC). Na maioria das fazendas do oeste catarinense, a atividade é secundária, com pequenos rebanhos, isto é, em média de 50 animais (Epagri, 2005). Dentre as limitações da expansão da atividade no estado de SC e nos demais estados do Brasil, a verminose é responsável por grandes perdas econômicas, relacionadas a perdas de apetite, desnutrição e consequentemente atrasos no crescimento, assim como morte dos animais não tratados (Thomaz-Soccol et al., 2004).

As verminoses nos rebanhos ovinos estão cada vez mais frequente e os tratamentos realizados apresentam na maioria dos casos baixa eficácia, isso devido os casos de resistência parasitária aos fármacos frequentemente usados. Várias são as causas da aceleração da resistência, dentre eles destacamos os erros de manejo e subdoses (Amarante et al., 1992). Em função da maioria dos ovinos ser criados no sistema a pasto, e muitas vezes em regiões úmidas ou mudanças climáticas podem favorece um ambiente favorável a sobrevivência de helmintos na sua forma infectante (Souza, 1997; Mascoma et al., 2008). Dentre os principais parasitos de ovinos, o Haemonchus contortus é o principal em questão de prevalência e patogenicidade, porém outras espécies de helmintos também são frequentes como Trichostongylus colubriformis, Cooperia spp., Teladorsagia spp., Oesophagostomum spp e Strongyloides spp (Waller, 1997). O Haemonchus spp. é o nematódeo que apresenta maior número de relatos de ocorrência em rebanhos ovinos no sul do Brasil, principalmente nas épocas mais quentes (Bricarello et al., 2004), diferente do Trichostrongylus spp. e Teladorsagia spp. que apresentam maior incidência nas épocas mais frias (Paolini et al., 2003).

Grande parte dos nematódeos gastrointestinais adquiriram resistência as principais classes de antiparasitários usados no combate a esses parasitos. Nos últimos anos, o monepantel tem sido a solução eficaz em fazendas no controle de nematódeos gastrointestinais (Ciuffa et al., 2013), porém na região oeste ele vem sendo pouco usado devido ao seu valor comercial elevado, diferente do levamisol que é um dos antiparasitários mais usados nos estados brasileiros. No entanto, existe relatos de resistência parasitaria ao monepantel para Haemonchus spp. descritas na Holanda (Van Den Brom et al., 2015), no Uruguai (Mederos et al., 2014) e no Brasil (Martins et al., 2017). Diante disse, o objetivo do presente estudo é avaliar a eficácia do monepantel e levamisol contra nematódeos gastrointestinais em ovinos naturalmente infectados. Um segundo objetivo seria verificar se as fezes desses animais usadas como adubação orgânica teria efeito tóxico a organismos da fauna do solo.

\section{MATERIAL E METODOS}

\section{ETAPA I}

Cinco fazendas de ovinos identificadas como A, B, C, D e E localizadas na região Oeste de Santa Catarina foram incluídas na primeira etapa desse estudo. Em um primeiro momento houve a formação de três grupos homogêneos (K, L, M) com 8 animais cada, sendo que os animais do grupo $\mathrm{K}$ foram medicados com monepantel na dose $2,5 \mathrm{mg} / \mathrm{kg}$, os ovinos do grupo $\mathrm{L}$ foram medicados com levamisol (Ripercol®) na dose de 5,0 mg/kg e os ovinos do grupo $\mathrm{M}$ foram usados como grupo controle (sem tratamento). Os dois antiparasitários foram administrados via oral, seguindo as doses recomendada pelo fabricante conforme descrito previamente nessa seção. Amostras de fezes foram coletadas diretamente da ampola retal no início do experimento (dia 0) e ao final do experimento (10 dias após tratamento). As amostras coletadas foram armazenadas em sacos plásticos, identificadas e separadas e foram levadas até o laboratório sobre refrigeração.

As fezes foram analisadas pela técnica de McMaster a fim de quantificar o número de ovos por grama de fezes (OPG) (Gordon E Whitlock, 1939). O cultivo de larvas foi realizado conforme descrito por Roberts \& O'Sullivan (1950), a fim de identificar os gêneros de helmintos envolvidos no parasitismo.

Os dados obtidos foram tabulados, posteriormente foram submetidos ao teste de normalidade. Os dados não apresentaram normalidade, então foram transformados para logaritmo, e em seguida submetidos ao teste de variância, seguido do teste de Duncan $(P$ $<0,05)$. O cálculo da eficácia do tratamento anti-helmíntico pode ser feito por meio do programa RESO 2.0 modificado utilizando a equação descrita a seguir:

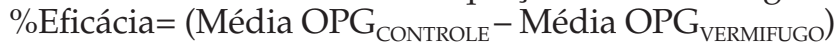
/ Média OPG Controle $^{*} 100$.

\section{ETAPA II}

Em uma segunda etapa do estudo, em uma fazenda foi formado os três grupos, descritos anteriormente, usando mesmos fármacos e doses do grupo controle, grupo de animais tratados com monepantel e grupo de animais tratados com levamisole (grupos K, Le M). O objetivo foi avaliar impactos ambientais caso seja usado as fezes desses animais que receberam antiparasitário para adubação orgânica. Após o quinto dia da administração do antiparasitário foi feita a coleta do material fecal para realização dos testes ecotoxicológicos. Nesse estudo usamos como marcador biológico o colêmbolo (Folsomia candida) e testes de sobrevivência e reprodução seguindo as metodologias (ISO, 2005) e ISO 11267 (ISO, 1999), respectivamente. Os exemplares de F. candida utilizados nos testes foram obtidos de criação já estabelecida no Laboratório de Solos e Sustentabilidade da UDESC-Oeste, sendo mantidos de acordo com diretrizes estabelecidas pela ISO 112682 (ISO, 1998). Para o teste de toxicidade crônica de F. candida, as recomendações seguidas foram de acordo com a ISO 11267 (ISO, 1999).

O experimento foi montado em recipientes de plástico com altura de $11,5 \mathrm{~cm}$ e diâmetro de $3,5 \mathrm{~cm}$ foram adicionados $30 \mathrm{~g}$ de solo com umidade corrigida para $60 \%$ da capacidade máxima de retenção de água (CRA). Cada unidade experimental recebeu 10 indivíduos com idade sincronizada entre 10 e 12 dias e mantidos em ambiente com temperatura de $20{ }^{\circ} \mathrm{C}+$ 2 e fotoperíodo de 12:12 (luz: escuro), semanalmente eles foram alimentados com $2 \mathrm{mg}$ de Saccharomyces 
cerevisiae, os recipientes foram abertos para promover a aeração e tiveram a umidade corrigida por diferença de peso. Após 28 dias do início do teste foi realizada a contagem do número de indivíduos jovens gerados. Para tanto adicionou-se água e tinta preta (para caneta esferográfica) aos recipientes. Por flutuação e com o contraste da tinta com os organismos foi possível fotografar as amostras e com auxílio do programa computacional ImageTool realizou-se a contagem.

A dose recomenda de fezes por hectare foi calculada com base no manual de adubação e calagem (CQFS RS/SC, 2004) para adubação orgânica sendo essa de 4,0 ton/ha, baseado nisso, definimos as doses de $0 ; 2$; 4 ; e 8 ton/ha de dejeto de ovinos. Para o desenvolvimento dos ensaios utilizou-se Solo Artificial Tropical (SAT) que consiste em uma mistura de $75 \%$ de areia fina, 20\% de caulin e 5\% de fibra de coco finamente moída (GARCIA, 2004). O solo utilizado teve a umidade corrigida para $65 \%$ da capacidade máxima de retenção de água e $\mathrm{pH}$ corrigido para 6,0 $\pm 0,5 \operatorname{com} \mathrm{CaCO}_{3}$, para todas as doses testadas foram utilizadas cinco réplicas.

No teste de sobrevivência e reprodução de F. candi$d a$, a diferenças entre a dose e o controle foram avaliadas através de análise de variância (ANOVA One-way) seguida pelo teste de Dunnet, utilizando Software Statistica 7.0

\section{RESULTADOS}

Houve uma redução do OPG nos animais tratados com monepantel $(\mathrm{P}<0.05)$ quando comparado com os demais tratamentos, conforme pode se observar na Tabela I. Em apenas uma fazenda, a redução de OPG foi inferior $(\mathrm{P}<0,05)$ nos animais tratados com levamisol comparado ao grupo controle. De acordo com a técnica de coprocultura, os grupos tratados com monepantel foi observado a resistência do Trichostrongylus spp. ao fármaco, já o Haemonchus spp. foi resistente ao levamisol na maioria das fazendas (Tabela II). A eficácia do monepantel nas fazendas A, B, C, D e E foi de 97, 98, 99, 100 e 100\%, respectivamente. Já, a eficácia do levamisol nas fazendas A, B, C, D e E foi de 69, -25, 52, 90 e $50 \%$, respectivamente (Figura 1).

O teste de sobrevivência e reprodução apresentou todos os critérios de validade, sendo que a mortalidade no controle foi inferior a $10 \%$ e o coeficiente de variação não ultrapassou 30\%. Verificamos que não houve diferença entre grupos na sobrevivência e reprodução de colêmbolos independente da dose usada para adubar o solo (Figura 2). Portanto os dois fármacos excretados nas fezes não causam efeito negativo a fauna de colembolos no solo.

\section{DISCUSSÃO}

No presente estudo o monepantel se mostrou eficaz em todas as fazendas, provavelmente porque essa droga tem mostrado resultados satisfatórios para helmintos multirresistentes (Sager et al., 2012). A eficácia do anti-helmíntico foi maior comparado com os grupos tratados com levamisol, onde apenas uma fazenda o anti-helmíntico teve sucesso. Isso pode ser dado em função do uso continuo do levamizole em muitas fazendas brasileiras e pelo fato do monepantel ser uma droga nova na região e pouco utilizada no combate a parasitoses. No entanto, existe muito variação de resultados entre regiões brasileiras, pois delineamento similar ao presente estudo foi feito em Minas Gerais, e os autores não constataram ineficácia do levamizole aos helmintos gastrointestinais, assim como baixa eficácia do monepantel ao Oesophagostomum spp. foi verificado por Ciuffa et al. (2017).

Os casos de resistência dos helmintos do gênero Trichostrongylus, tem grande prevalência em fazendas de produção de pequenos ruminantes, e já vem sendo comprovada na maioria das classes de anti-helmínticos como ivermectina, moxidectina, albendazol, levamisol, closantel e trichlorfon (Almeida et al., 2010; Chandrawathani et al., 2013). Em estudo no Brasil, utilizando monepantel como anti-helmíntico, foi evidenciado a ineficácia contra T. colubriformis, no entanto, contra T. axei, o tratamento se mostrou eficaz, revelando a existência de diferenças entre espécies do

Tabela I. Número médio de ovos por grama de fezes (OPG), presentes nas amostras de fezes de ovinos naturalmente infectados antes e depois dos respectivos tratamentos (Average number of eggs per gram of feces (OPG), present in fecal specimens of naturally infected ewes before and after the respective treatments.)

\begin{tabular}{|c|c|c|c|c|c|}
\hline \multirow{2}{*}{ Propriedade } & \multirow{2}{*}{ Tratamento } & \multicolumn{3}{|c|}{ Médias de OPG } & \multirow{2}{*}{$P$} \\
\hline & & Controle & Levamisol & Monepantel & \\
\hline \multirow{2}{*}{ K } & Antes & $1487,5 \pm 2464$ & $912,5 \pm 1257$ & $1612,5 \pm 2457$ & $P>0,05$ \\
\hline & Depois & $1775 \pm 1917^{a}$ & $550 \pm 515^{a}$ & $50 \pm 141^{b}$ & $P<0,05$ \\
\hline \multirow{2}{*}{ L } & Antes & $2550 \pm 1582$ & $4325 \pm 3699$ & $4050 \pm 2538$ & $P>0,05$ \\
\hline & Depois & $3100 \pm 2675^{a}$ & $3887,5 \pm 3749^{a}$ & $75 \pm 116^{b}$ & $P<0,05$ \\
\hline \multirow{2}{*}{ M } & Antes & $137,5 \pm 130$ & $362,5 \pm 573$ & $400 \pm 393$ & $P>0,05$ \\
\hline & Depois & $1025 \pm 1493^{a}$ & $487,5 \pm 996^{a}$ & $12,5 \pm 35^{b}$ & $P<0,05$ \\
\hline \multirow{2}{*}{$\mathrm{N}$} & Antes & $5112,5 \pm 6669$ & $1250 \pm 1608$ & $3462,5 \pm 4419$ & $P>0,05$ \\
\hline & Depois & $3662,5 \pm 2154^{a}$ & $350 \pm 228^{b}$ & $12,5 \pm 35^{c}$ & $P<0,05$ \\
\hline \multirow{2}{*}{$\mathrm{O}$} & Antes & $587,5 \pm 700$ & $2225 \pm 4065$ & $1312,5 \pm 2552$ & $P>0,05$ \\
\hline & Depois & $450 \pm 687^{a}$ & $225 \pm 483^{a}$ & $0 \pm 0^{\mathrm{b}}$ & $P<0,05$ \\
\hline
\end{tabular}

$\mathrm{P}<0,05$, com letras diferentes na mesma linha mostra diferença entre grupos no teste Duncan. 
Tabela II. Porcentagem de helmintos (Haemonchus [HAE], Trichostrongylus [TRI], presentes nas amostras fecais dos grupos tratados com os fármacos (Levamisol e Monepantel) (Percentage of helminths (Haemonchus [HAE], Trichostrongylus [TRI], present in fecal specimens from drug-treated groups (Levamisole and Monepantel).

\begin{tabular}{lccccccc}
\hline & & \multicolumn{4}{c}{ Porcentagem helmintos por grupo } \\
\cline { 3 - 7 } Propriedade & Tratamento & \multicolumn{2}{c}{ Grupo Controle } & Tratamento-Monepantel & \multicolumn{2}{c}{ Tratamento-Levamisol } \\
\cline { 3 - 7 } & & HAE & TRI & HAE & TRI & HAE & TRI \\
\hline \multirow{2}{*}{ A } & Antes & 30 & 70 & 32 & 68 & 33 & 67 \\
& Depois & 20 & 80 & 90 & 10 & 85 & 15 \\
B & Antes & 95 & 5 & 93 & 7 & 86 & 14 \\
& Depois & 86 & 14 & 41 & 59 & 92 & 8 \\
C & Antes & 89 & 11 & 34 & 66 & 75 & 25 \\
& Depois & 78 & 22 & 25 & 75 & 78 & 22 \\
D & Antes & 60 & 40 & 68 & 32 & 66 & 34 \\
& Depois & 62 & 38 & 30 & 70 & 30 & 70 \\
E & Antes & 82 & 18 & 88 & 83 & 72 & 28 \\
\end{tabular}

gênero na questão de resistência parasitária (Cintra et al., 2016). Outros estudos realizados na Nova Zelândia apresentaram resistência do T. colubriformis aos fármacos albendazol e/ou levamisol, já o T. axei demonstrou resistência apenas ao levamisol (Waghorn et al., 2014). Na Austrália, estudo mostrou resistência de Haemonchus spp. ao monepantel e eficácia reduzida de uma combinação de derquantel/abamectina em ovelhas (Sales; Love 2016). Pesquisadores australianos também concluíram que devido ao amplo espectro de resistência é necessário a integração de métodos alternativos sustentáveis no controle do parasito, a fim de retardar o desenvolvimento da resistência e aumentar a eficácia da vida dos anti-helmínticos (Lamb et al., 2017).

O levamisol pertencente a classe dos imidazotiazóis, é amplamente utilizado no combate aos nematódeos gastrointestinais, em função do uso excessivo na maioria dos casos, o mesmo apresenta baixa eficácia na eliminação de formas imaturas (Vieira et al., 1989). Além de contribuir para geração de populações de nematódeos resistentes a utilização do levamisol e outros fármacos pertencente à família dos imidazotiazóis. De acordo com Echevarria et al. (1996), 80\% dos rebanhos do Rio Grande do Sul apresentaram resistência a ad-

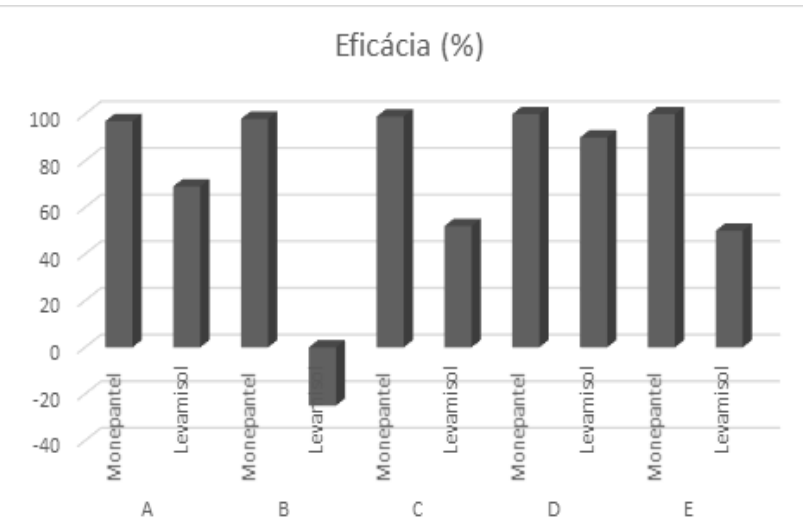

Figura 1. Eficácia dos tratamentos anti-helmíntico nas propriedades testadas (Efficacy of anthelmintic treatments on proven properties). ministração do levamisol em seus rebanhos. O gênero Haemonchus na maioria dos casos de helmintoses é o que apresenta maior resistência (Vieira et al., 2014). Grande resistência que pode ser explicada por possui alta variabilidade genética e altas taxas de mutação em conjunto com prolificidade (Melo \& Bivilaquia, 2005).

As drogas testadas não apresentaram nenhum efeito maléfico sobre a fauna edáfica do solo, assim eliminando o risco de prejudicar os organismos vivos após sua excreção no ambiente. Conhecendo a grande importância dos ensaios ecotoxicologicos que tem por finalidade investigar os efeitos do tratamento realizado sobre o organismo do solo (Brentano, 2006). Importante a escolha das melhores espécies para indicar a toxicidade de determinados produtos químicos, como é o caso da espécie de colembolos ( $F$. candida), espécie sensível a contaminantes e com boas taxas de reprodução e fácil de manter em laboratório (Fountain \& Hopkin, 2005).

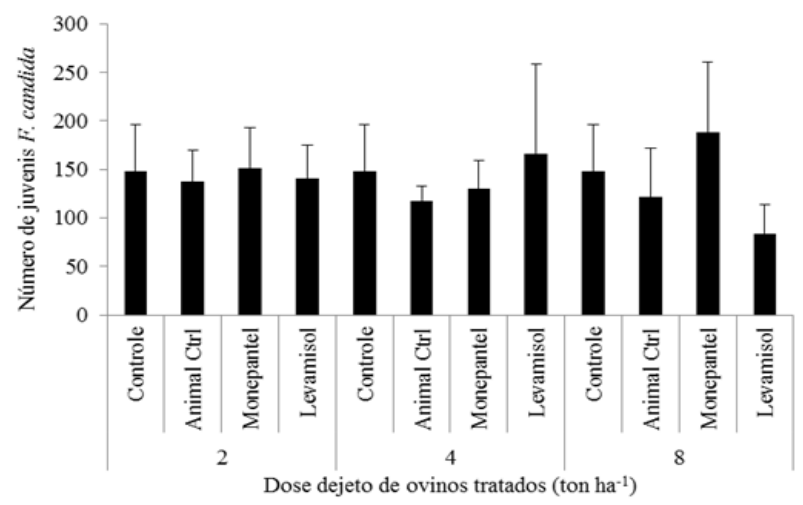

Figura 2. Efeito de antiparasitários nas diferentes doses de dejetos em relação ao número de juvenís $F$. candida. Não houve diferença entre doses e fármacos, isto é, as fezes dos animais tratados não tiveram efeito ecotoxicologico sobre colembolos (Effect of antiparasitic agents in different doses of manure in relation to the number of juveniles $F$. Candida. There was no difference between doses and drugs, i.e. the feces of treated animals had no ecotoxicological effect on springtails). 
A fauna edáfica do solo atua como prestadora de diversos serviços ecossistêmicos (Baretta, 2011), trazendo benefícios para o meio ambiente, portanto é de extrema importância no monitoramento do efeito que os fármacos exercem sobre a mesma, em função do uso de drogas em larga escala com proposito de controlar os problemas causados pelos helmintos gastrointestinais. Importante ressaltar que estudo recente mostrou que a uso de quatro produtos antiparasitários (ivermectina, fipronil, fluazuron e closantel) interferem na sobrevivência ou reprodução de colembolos (Zortea et al., 2017). O uso do monepantel em combate aos helmintos, vem ganhando bastante espaço nas fazendas criadoras de ovinos em função de sua eficácia comprovada, mas ainda existem poucos estudos na literatura sobre seus efeitos no solo quando excretado na urina e fezes (Stuchlíková et al., 2014). Porém, nesse estudo verificamos que não interfere na reprodução dos colembolos.

Baseado nos resultados, nos concluímos que o uso do monepantel no combate aos helmintos gastrointestinais foi eficaz na redução da quantidade de OPG, sendo recomendado o uso em fazendas que enfrentam sérios problemas com resistência parasitária. $\mathrm{O}$ levamisol não foi eficaz, pois é muito utilizado em fazendas com problemas de verminoses. $\mathrm{O}$ uso do medicamento não influenciou negativamente sobre a fauna edáfica do solo através das excretas dos animais tratados, ficando claro que o mesmo não oferece risco a população de organismos do solo, eliminando as chances de poluição ambiental.

\section{REFERENCIAS}

Almeida, F.A., Garcia, K.C.O.D., Torgerson, P.R., \& Amarante, A.F.T.D.

(2010). Multiple resistance to anthelmintics by Haemonchus contortus and Trichostrongylus colubriformis in sheep in Brazil. Parasitology international, 59, 622-625.

Amarante, A.F.T., Barbosa, M.A., de Oliveira, M.A.G., Carmello, M.J. \& Padovani, CR. (1992). Efeito da administração de oxfendazol, ivermectina e levamisol sobre os exames coproparasitológicos de ovinos. Brazilian Journal of Veterinary Research and Animal Science, 29, 31-38.

Baretta, D., Santos, J.C.P., Segat, J.C., Geremia, E.V., Oliveira Filho, L.C.I. \& Alves, M.V. (2011). Fauna edáfica e qualidade do solo, In: Tópicos em Ciência do Solo. Viçosa: Sociedade Brasileira de Ciência do Solo, 7, 119-170.

Brentano, D.M. (2006). Desenvolvimento e aplicação do teste de toxicidade crônica com Daphnia magna: avaliação de efluentes tratados de um aterro sanitário.

Bricarello, P.A., Gennari, S.M., Oliveira-Sequeira, T.C.G., Vaz, C.M.S.L., De Gonçalves, I.G. \& Echevarria, F.A.M. (2004). Worm burden and immunological responses in Corriedale and Crioula Lanada sheep following natural infection with Haemonchus contortus. Small Ruminant Research, 51, 75-83.

Chandrawathani, P., Premaalatha, B., Nurulaini, R., Erwanas, A.I., Zaini, C.M., Aizan, M. \& Khadijah, S. (2013). Severe anthelmintic resistance in two free grazing small holder goat farms in Malaysia. Journal Veterinary Science Technology, 4, 2.

Ciuffa, A. Z., Urzedo, M. C. C., Silva, D. M., Pires, B. C., Rezende, L. M., Brasão, S. C., Rosalinski-Moraes, F. (2017). Effectiveness of monepantel and levamisole to control gastrointestinal strongylid parasites of sheep. Bioscience Journal, 33(3), 639-643.
Cintra, M.C.R., Teixeira, V.N., Nascimento, L.V. \& Sotomaior, C.S. (2016). Lack of efficacy of monepantel against Trichostrongylus colubriformis in sheep in Brazil. Veterinary Parasitology, 216, 4-6.

Echevarria, F., Borba, M.F.S., Pinheiro, A.C., Waller, P.J. \& Hansen, J.W. (1996). The prevalence of anthelmintic resistance in nematode parasites of sheep in Southern Latin America: Brazil. Veterinary Parasitology, 62, 199-206.

Epagri. (2005), Cadeia produtiva de ovinos no Estado de Santa Catarina. Florianópolis: Epagri.

Fountain, M.T. \& Hopkin, S.P. (2005). Folsomia candida (Collembola): a "standard" soil arthropod. Annual Review Entomology, 50, 201-222.

Garcia, M.V. (2004). Effects of pesticides on soil fauna: development of ecotoxicological test methods for tropical regions. Ecology and Development Series, 19, 281-287.

Gordon, H.M. \& Whitlock, H.V. (1939). A new technique for counting nematode eggs in sheep faeces. Journal of the council for Scientific and Industrial Research, 12, 50-52.

ISO, SQ. (1999). Inhibition of Reproduction of Collembola (Folsomia candida) by Soil Pollutants, no. 11267, ISO, Geneva.

ISO, SQ. (1998). Soil quality: Effects of pollutants on earthworms (Eisenia fetida). Part 2: Determination of effects on reproduction, ISO 11268-2 Geneva.

ISO, SQ. (2005). Avoidance test for testing the quality of soils and effects of chemicals on behavior - Part 2: Test with collembolan (Folsomia candida). ISO 17512-2 Geneve, Switzerland.

Lamb, J., Elliott, T., Chambers, M., Chick, B. (2017) Broad spectrum anthelmintic resistance of Haemonchus contortus in northern NSW of Australia. Veterinary Parasitology, 241, 48-51.

Martins, A.C., Bergamasco, P.L.F., Felippelli, G., Tebaldi, J.H., Duarte, M.M.F., Testi, A.J.P., Lapera, I.M., Hoppe, E.G.L. (2017) Haemonchus contortus resistance to monepantel in sheep: fecal egg count reduction tests and randomized controlled trials. Semina: Ciências Agrarias 38, 231-238

Mas-Coma, S., Valero, M. A., Bargues, M. D. (2008). Effects of climate change on animal and zoonotic helminthiases. Revue Scientifique et Technique (International Office of Epizootics), 27, 443-457.

Medeiros, A.E., Ramos, Z. \& Banchero, G.E. (2014). First report of monepantel Haemonchus contortus resistance on sheep farms in Uruguay. Parasites \& vectors, 7, 598-599.

Melo, A.C.F.L., Bevilaqua, C.M.L. (2005). Abordagem genética da resistência anti-helmíntica em Haemonchus contortus Genetic approach of anthelmintic resistance in Haemonchus contortus, Revista Portuguesa de Ciências Veterinárias, 100, 141-146.

Paolini, V., Frayssines, A., De La Farge, S., Dorchies, P., Hoste, H (2003). Effects of condensed tannins on established populations and in incoming larvae of Trichostrongylus colubriformis and Teladorsagia circumcincta in goats, Veterinary Research, 34, 331-339.

Roberts, F.H.S. \& O'Sullivan, P.J. (1950). Methods for egg counts and larval cultures for strongyles infesting the gastro-intestinal tract of cattle. Australian Journal of Agricultural Research, 1, 99-102.

Sager, H., Bapst, B., Strehlau, G.A. \& Kaminsky, R. (2012), Efficacy of monepantel, derquantel and abamectin against adult stages of a multi-resistant Haemonchus contortus isolate. Parasitology research, $111,2205-2207$

Sales, N., Love, S. (2016) Resistance of Haemonchus sp. to monepantel and reduced efficacy of a derquantel/abamectin combination confirmed in sheep in NSW Australia. Veterinary Parasitology, 228, 193-196.

Souza, F.P.D. (1997). Contribuição para o estudo da resistencia de helmintos gastrointestinais de ovinos [Ovis aries] a anti-helminticos, no estado do Parana. Dissertação. Programa de Pós-graduação em Ciências Veterinárias, Universidade Federal do Paraná.

Stuchlíková, L., Jirásko, R., Vok冈ál, I., Valát, M., Lamka, J., Szotáková, B., Skálová, L. (2014). Metabolic pathways of anthelmintic drug monepantel in sheep and in its parasite (Haemonchus contortus). Drug Testing and Analysis, 6(10), 1055-1062.

Thomaz-Soccol, V., Souza, F.P.D., Sotomaior, C., Castro, E.A., Milczewski, V. \& Mocelin, G. (2004). Resistance of gastrointestinal nematodes to 
anthelmintics in sheep (Ovis aries). Brazilian Archives of Biology and Technology, 47, 41-47.

Van den Brom, R., Moll, L., Kappert, C. \& Vellema, P. (2015). Haemonchus contortus resistance to monepantel in sheep. Veterinary Parasitology, 209, 278-280.

Vieira, L.D.S., Berne, M., Cavalgante, A. \& Menezes, R. (1989). Redução do número de ovos por grama de fezes (OPG) em caprinos e ovinos medicados com anti-helmínticos. Embrapa Caprinos e Ovinos-Boletim de Pesquisa e Desenvolvimento (INFOTECA-E).

Vieira, L.S., Osório, J.C.S., Selaive, A.B. (2014). Doenças parasitárias em ovinos: Produção de ovinos. São Paulo: Roca, 311-342.
Waghorn, T.S., Knight, J.S. \& Leathwick, D.M. (2014). The distribution and anthelmintic resistance status of Trichostrongylus colubriformis, $T$. vitrinus and T. axei in lambs in New Zealand. New Zealand Veterinary Journal, 62, 152-159.

Waller, P.J. (1997). Anthelmintic resistance. Veterinary Parasitology, 72, 391-412.

Zortéa, T., Segat, J.C., Maccari, A.P., Sousa, J.P., Da Silva, A.S., Baretta, D. (2017). Toxicity of four veterinary pharmaceuticals on the survival and reproduction of Folsomia candida in tropical soils. Chemosphere, 173, 460-465. 